\title{
Nest desertion: a trade-off between current and future reproduction
}

\author{
NANETTE VERBOVEN* \& JOOST M. TINBERGEN† \\ *Netherlands Institute of Ecology, Heteren \\ $\dagger$ Department of Animal Ecology, University of Groningen \\ (Received 25 January 2001; initial acceptance 11 May 2001; \\ final acceptance 29 November 2001; MS. number: 6812)
}

\begin{abstract}
Life history theory predicts that parents should desert a reproductive attempt if the costs of rearing this brood exceed the expected benefits. Thus, if the value of the current breeding attempt is reduced, for example through an unexpected reduction in size, parents are expected to reconsider whether it is worth continuing investing in their brood. With regard to nest desertion behaviour two predictions can be made: individuals are (1) more likely to desert if the reduction in clutch size is large and (2) less likely to desert if the reduction is at a late stage of breeding. We investigated the threshold at which nest desertion takes place by experimentally reducing great tit, Parus major, clutches to different sizes and at different stages of the incubation period. The results were in accordance with the predictions: clutch desertion rates were negatively related to the number of eggs that remained in the nest, and nest desertion was less likely nearer the end of the incubation period. In addition, we estimated the fitness consequences of nest desertion behaviour. For this purpose we made one group of birds desert in favour of a replacement clutch and another group rear a reduced brood. The latter were more likely to produce a second clutch after the first-brood fledglings had left the nest. As a consequence, the number of fledglings produced over the entire breeding season did not differ between the two experimental groups. We also counted the number of recruits and breeding adults in the following breeding season and found that the experimental groups did not differ in local recruitment and adult survival. Therefore, the results did not indicate that parents improved their fitness by deserting a reduced clutch.
\end{abstract}

๑ 2002 The Association for the Study of Animal Behaviour. Published by Elsevier Science Ltd. All rights reserved.

One of the major trade-offs in life history theory is concerned with the division of reproductive effort between current and future breeding attempts and is also termed the cost of reproduction (Williams 1966). In making parental investment decisions, iteroparous species are assumed to take into account the benefit from the current brood in relation to the fitness costs in terms of future broods. This implies that parents are not expected to embark on a reproductive attempt if this will result in a net loss of fitness.

Life history theory often addresses questions such as how many eggs should be laid. Indeed several studies have shown that birds tend to lay the clutch size that maximizes fitness (Pettifor et al. 1988; Daan et al. 1990; Tinbergen \& Daan 1990). However, if circumstances change after the clutch has been laid, particularly if the

Correspondence and present address: N. Verboven, Ornithology Group, Division of Environmental and Evolutionary Biology, Graham Kerr Building, University of Glasgow, Glasgow G12 8QQ U.K. (email: nv6r@udcf.gla.ac.uk).J. M. Tinbergen is at the Department of Animal Ecology, University of Groningen, P.O. Box 14, 9750 AA Haren, The Netherlands. value of the current breeding attempt is reduced through an unexpected reduction in clutch size, parents have to reconsider whether it is worth continuing investing in the current breeding attempt (Lessells 1993). If this is not the case, parents are expected to stop investment in that brood. Desertion of breeding attempts has indeed been recorded in naturally (Mock \& Parker 1986; Fernández \& Reboreda 2000) and experimentally reduced broods (Winkel 1970; Armstrong \& Robertson 1988; Beissinger 1990; Winkler 1991; Delehanty \& Oring 1993; Bauchau \& Seinen 1997). Two predictions can be made with regard to the relationship between clutch size reduction and the occurrence of nest desertion.

The first prediction is that birds are more likely to desert if the reduction in clutch or brood size is large. This is because a larger reduction will result in a smaller benefit from the current breeding attempt, while the cost in terms of future broods is unlikely to change, because, in terms of time, comparable amounts of parental investment are required to complete a reduced or a full-sized breeding attempt. 
The second prediction is that birds are less likely to desert if the clutch size reduction occurs later rather than earlier during the breeding attempt. At the termination of parental care the value of a reduced brood is likely to be the same irrespective of the time at which the reduction took place. However, the remaining investment parents have to make to rear the offspring to independence declines with the stage at which the reduction occurs. Towards the end of the period of parental care the fitness value of the nest increases relative to the fitness value of the parents. Therefore, nest desertion is expected to become less likely at later stages of breeding. However, an alternative explanation for such a relationship is that accumulation of parental investment throughout the nesting cycle affects the probability of nest desertion by increasing the cost of future reproduction (Slagsvold 1982).

To investigate the threshold at which nest desertion is expected, we should measure the fitness consequences of the two alternative decisions: continue with the current breeding attempt or desert it in favour of future reproduction.

Our aims in this study were twofold. First, we tested the two hypotheses and analysed nest desertion rates of experimentally reduced nests in relation to the size and the stage of the current breeding attempt. Second, we attempted to measure the fitness consequences of investment in a reduced clutch on the one hand and investment in future reproduction on the other. We used these values to explain the occurrence of nest desertion in relation to the size of the reduced clutch.

We conducted two separate sets of experiments: one to measure desertion rates and one to measure fitness consequences. Nest desertion rates were studied by means of egg removal experiments in which the birds had the choice either to desert or to stay with a reduced clutch ('free choice' experiments). Fitness estimates on the other hand were obtained from birds that were made to desert the current clutch or that were manipulated in such a way that they continued with a reduced clutch. By creating a 'no choice' group we made sure that birds of different quality, possibly resulting in different nest desertion behaviour, were equally represented in the two categories of fitness measurements.

\section{METHODS}

\section{Nest Desertion Groups}

To test whether nest desertion probabilities were related to the size of the current clutch (prediction 1) we reduced clutches of great tits, Parus major, to two different sizes. To see how many eggs we had to remove to obtain measurable desertion rates, we explored data collected in 1960-1963 as part of an experimental study on population regulation (Kluyver 1971). We decided to reduce clutches to three and to five eggs 8 days after the start of incubation. By doing so we expected an average desertion rate of approximately $50 \%$ and a clear response to the experimental treatments. The experiment was performed in 3 years (1993-1995).
To study the relationship between nest desertion probability and the stage of the breeding attempt (prediction 2), we reduced clutches at different stages of the incubation period. In 1995 we reduced clutches to five eggs on day 4 of incubation and to three eggs on day 12. We did not reduce clutches to three eggs at day 4 and to five eggs at day 12, because we expected higher and lower desertion rates, respectively, than those after a similar reduction at day 8 of incubation (i.e. probabilities approaching 1 and 0 ) and thus there would have been no clear response to the manipulation.

To investigate the possibility that desertion rates were related to the accumulation of costs during the breeding attempt, we studied the replacement clutches (clutches produced after an unsuccessful first clutch) of birds that had incubated the first clutch for a different length of time ( 4,8 or 12 days). We removed the rest of the clutch in the previous experiment after recording whether the birds had deserted. We then compared the replacement clutches of the birds that had incubated for 4 days or 12 days with replacement clutches that were produced after 8 days of incubation ('forced desertion', see below).

\section{Fitness Consequences Groups}

We created three additional experimental groups in 1995 to estimate the fitness consequences of desertion and continuation. In the first two groups we removed all but either three or five eggs after 8 days of incubation. To make nest desertion unlikely, we replaced the removed eggs with plastic dummy eggs ('forced continuation'), which were removed as soon as the rest of the clutch hatched. In the third group we removed all eggs at day 8 of incubation. These birds had no other option than to desert the current breeding attempt ('forced desertion').

\section{Experimental Procedure}

We studied a population of individually ringed great tits on the island of Vlieland (see Kluyver 1971 and Verhulst \& van Eck 1996 for details of the study area). Every year all nestboxes on the island, including those in the village, were checked at least once per week to identify breeding birds and to determine the laying date and size of the clutch. The length and the breadth of the eggs were measured to the nearest $0.1 \mathrm{~mm}$ and egg volume was calculated using the formula in van Noordwijk et al. (1981). The incubation period is 14 days. Since great tits usually start incubation as soon as the clutch is complete, we took the day the last egg was laid as day 0 of incubation. Nestlings were ringed and measured when they were 15 days old. Body mass at day 15 was taken as fledging mass.

We assigned nests to different experimental treatments by matching laying date ( \pm 1 day) and clutch size $( \pm 1$ egg). For each set of experimental treatments we assigned one (1993), two (1994) or three (1995) nests to an unmanipulated group, also matching laying date and clutch size. In the unmanipulated group no eggs were removed. 
Table 1. Laying date (April) and original clutch size of experimental great tit clutches used to measure desertion rates in relation to manipulated clutch size and stage of the incubation period at the time of manipulation

\begin{tabular}{|c|c|c|c|c|c|c|c|c|c|c|}
\hline \multirow{2}{*}{$\begin{array}{l}\text { No. of eggs after } \\
\text { manipulation }\end{array}$} & \multirow{2}{*}{$\begin{array}{l}\text { Stage of } \\
\text { incubation }\end{array}$} & \multicolumn{3}{|c|}{1993} & \multicolumn{3}{|c|}{1994} & \multicolumn{3}{|c|}{1995} \\
\hline & & Laying date & Clutch size & $N$ & Laying date & Clutch size & $N$ & Laying date & Clutch size & $N$ \\
\hline Unmanipulated & & $26.69 \pm 3.06$ & $8.91 \pm 1.31$ & 32 & $28.33 \pm 2.61$ & $8.64 \pm 1.47$ & 36 & $26.56 \pm 3.22$ & $8.86 \pm 1.16$ & 36 \\
\hline 5 eggs & Day 4 & - & - & - & - & - & - & $26.6 \pm 4.53$ & $9.10 \pm 1.44$ & 10 \\
\hline 5 eggs & Day 8 & $26.59 \pm 3.09$ & $8.83 \pm 1.29$ & 29 & $28.61 \pm 3.06$ & $8.57 \pm 1.47$ & 23 & $26.50 \pm 3.33$ & $8.58 \pm 0.64$ & 12 \\
\hline 3 eggs & Day 8 & $26.62 \pm 3.07$ & $8.90 \pm 1.30$ & 29 & $28.42 \pm 2.77$ & $8.67 \pm 1.21$ & 24 & $26.58 \pm 3.50$ & $9.08 \pm 0.95$ & 12 \\
\hline 3 eggs & Day 12 & - & - & - & - & - & - & $26.4 \pm 3.75$ & $8.60 \pm 1.07$ & 10 \\
\hline
\end{tabular}

Means are given \pm SD. Four clutches in the 5-egg group and three clutches in the 3-egg group were deserted before the female was identified. These clutches are not included in the table and are excluded from the analyses of nest desertion.

To make sure that nest space was available for renesting in case of nest desertion, we provided an empty nestbox in each territory where we planned to remove eggs. This nestbox was put up in a tree less than $10 \mathrm{~m}$ away from the occupied nestbox, 2 days before the experiment. On the day before egg removal, or 7 days after the start of incubation, the incubating female was identified. We did this by reading the colour ring combination, or the number on the metal ring, by carefully pushing the bird to one side while she remained sitting on the nest. In the few cases in which the female was not ringed, we marked her with red nail varnish on the outer tail feathers, again without taking her from the nest. Identification of the female never caused nest desertion. Before we removed eggs from the clutch, we observed the nestbox from a distance until the female was seen to leave. Eggs were removed under licence of the Animal Experimentation Committee of the Royal Dutch Academy of Arts and Sciences (KNAW), stored at $-20^{\circ} \mathrm{C}$ and later used for other purposes.

On the day after the manipulation we determined whether the clutch was deserted. We classified the nest as deserted if the female was absent and if the eggs were cold. Nests were always deserted within a day of egg removal. Replacement clutches were discovered during the weekly nestbox inspections. Great tits rarely switch mates between successive breeding attempts in one season (Kluyver 1951, personal observation); therefore abandoned and replacement clutches were matched using the identity of the female. If there was doubt about the identity of the female (e.g. faded red marks), we also used desertion and laying dates. The majority of females that deserted the first clutch were found renesting (91\% in the 'free choice' group and $100 \%$ in the 'forced desertion' group).

We obtained fitness estimates by recording the breeding success of the birds in the 'forced desertion' and the 'forced continuation' group. We counted the number of fledglings produced over the entire breeding season and recorded local recruitment and adult survival by determining the number of birds breeding in the study area in the next breeding season.

\section{Statistical Analysis}

Data were analysed with the GLIM statistical package (Numerical Algorithms Group 1993). Data on proportions such as nest desertion probability, fledging success (probability that a hatchling survived to fledging), probability of a second clutch (a clutch produced after a successful first brood), recruitment and adult survival were analysed with logistic regression (Sokal \& Rohlf 1995) by specifying binomial errors and logit link in GLIM. Because the number of recruits is not normally distributed (many pairs produce no recruits at all), we used Poisson errors to analyse the number of recruits (Crawley 1993). We used the change in deviance and the degrees of freedom to assess significance of the variables tested. Continuous variables, such as laying date, clutch size, number of fledglings and renesting interval (number of days between the date of egg removal and the laying date of the first egg of the replacement clutch) were analysed with normal errors and significance was assessed with $F$ tests. Differences between the experimental treatments were tested by introducing two factors with three levels each: one for clutch size (unmanipulated, three eggs, five eggs) and one for stage of incubation at the time of manipulation (day 4, 8, 12). All statistical tests were two tailed.

\section{RESULTS}

Before analysing desertion rates and fitness estimates we checked whether we had succeeded in assigning pairs equally to different groups with regard to laying date and clutch size. The unmanipulated group and the groups with three or five eggs did not differ in laying date (ANOVA, controlled for year: $F_{2,228}=0.01, P=0.98$ ) or original clutch size (ANOVA: $F_{2,230}=0.26, \quad P=0.77$; Table 1). Similar results were found for the clutches that were reduced at 4,8 and 12 days of incubation (ANOVA: laying date: $F_{2,41}=0.01, P=0.99$; clutch size: $F_{2,41}=0.67$, $P=0.52$; Table 1 ). When comparing the three groups that were used for fitness measurements we also did not find differences with regard to laying date (ANOVA: $F_{2,57}=0.01, P=0.99$ ) or original clutch size (ANOVA: $F_{2,57}=1.01, P=0.37$; Table 2). We conclude that birds with different laying dates and clutch sizes were equally distributed over the experimental groups.

We also checked whether we had succeeded in 'forcing' birds to continue with a reduced clutch by replacing removed eggs with dummy eggs. Birds with reduced clutches supplemented with dummy eggs were not more 
Table 2. Fitness estimates for great tit pairs with unmanipulated clutches, reduced clutches with dummy eggs ('forced continuation' group) and replacement clutches ('forced desertion' group)

\begin{tabular}{|c|c|c|c|c|c|c|c|c|c|c|c|}
\hline & Unmanipulated & $N$ & 5 eggs & $N$ & & 3 eggs & $N$ & & Replacement & $N$ & \\
\hline Laying date (April) & $26.56 \pm 3.26$ & 36 & $26.42 \pm 3.45$ & 12 & & $26.50 \pm 3.90$ & 12 & & $48.83 \pm 6.35$ & 12 & $C^{* *}$ \\
\hline Original clutch size & $8.86 \pm 1.17$ & 36 & $8.67 \pm 1.44$ & 12 & & $9.33 \pm 0.78$ & 12 & & $8.42 \pm 1.31$ & 12 & \\
\hline Proportion of nests fledged & 0.89 & 36 & 0.92 & 12 & & 0.83 & 12 & & 0.83 & 12 & \\
\hline Fledging success per hatchling† & 0.94 & 32 & 0.98 & 11 & $\mathrm{R}^{*}$ & 1.00 & 10 & $\mathrm{R}^{*}$ & 0.84 & 9 & $C^{*}$ \\
\hline No. of fledglings first brood $\dagger$ & $7.28 \pm 1.61$ & 32 & $4.73 \pm 0.90$ & 11 & $C^{\star *} R^{*}$ & $2.90 \pm 0.32$ & 10 & $C^{* *} R^{\star *}$ & $6.44 \pm 1.24$ & 9 & \\
\hline Fledging mass $(q) \dagger$ & $17.11 \pm 1.03$ & 32 & $17.96 \pm 0.45$ & 11 & $C^{*} R^{*}$ & $18.11 \pm 0.99$ & 10 & $C^{*} R^{*}$ & $16.78 \pm 1.31$ & 9 & \\
\hline Recruitment per fledgling $\dagger$ & 0.100 & 32 & 0.091 & 11 & & 0.033 & 10 & & 0.076 & 9 & \\
\hline No. of recruits first brood $\dagger$ & 0.66 & 32 & 0.45 & 11 & & 0.10 & 10 & $C^{*} R^{*}$ & 0.60 & 10 & \\
\hline Proportion of 2 nd broods $\dagger$ & 0.34 & 32 & 0.73 & 11 & $C^{*} R^{*}$ & 0.70 & 10 & $C^{*} R^{*}$ & 0.10 & 10 & \\
\hline Interbrood interval (days) & $4.27 \pm 3.44$ & 11 & $1.23 \pm 2.85$ & 8 & $\mathrm{C}^{*}$ & $0.29 \pm 3.40$ & 7 & $C^{*}$ & $7.00 \pm 0.00$ & 1 & \\
\hline No. of fledglings second brood $\dagger$ & $6.11 \pm 1.05$ & 9 & $5.50 \pm 1.23$ & 6 & & $5.86 \pm 1.07$ & 7 & & $5.00 \pm 0.00$ & 1 & \\
\hline No. of recruits second brood $\dagger$ & 0.00 & 9 & 0.33 & 6 & & 0.29 & 7 & & 0.00 & 1 & \\
\hline No. of fledglings per season & $8.03 \pm 4.70$ & 35 & $7.08 \pm 3.50$ & 12 & & $5.83 \pm 2.66$ & 12 & & $5.73 \pm 3.74$ & 11 & \\
\hline No. of recruits per season & 0.58 & 36 & 0.67 & 12 & & 0.25 & 12 & & 0.50 & 12 & \\
\hline Female survival & 0.31 & 35 & 0.33 & 12 & & 0.42 & 12 & & 0.50 & 12 & \\
\hline Male survival & 0.26 & 34 & 0.30 & 10 & & 0.50 & 10 & & 0.33 & 12 & \\
\hline
\end{tabular}

Means are given \pm SD. In two broods the number of fledglings was unknown and in one brood the nestlings were not weighed. One female and six males were not ringed; these birds are not included in the adult survival rates. C: significantly different from unmanipulated control clutches, R: significantly different from replacement clutches, ${ }^{*} P<0.05 ;{ }^{*} P<0.001$

$\dagger$ †roods from which at least one chick fledged.

likely to desert between day 8 and hatching than birds with unmanipulated clutches (chi-square test: $\chi_{2}^{2}=1.51$, $P=0.47)$. Thus birds in the 'forced continuation' group formed an unbiased sample with regard to desertion behaviour.

\section{Prediction 1: Desertion and Clutch Size}

Since clutch desertion rates did not differ between the three experimental years (chi-square test: $\chi_{2}^{2}=0.70$, $P=0.70$ ), we combined the results for 1993-1995. Overall $4.8 \%(N=104)$ of the unmanipulated clutches were deserted between day 8 of incubation and hatching (Fig. 1). This could be seen as natural desertions arising from, for example, accidental death of one of the parents. Clutches reduced to five eggs were deserted more often

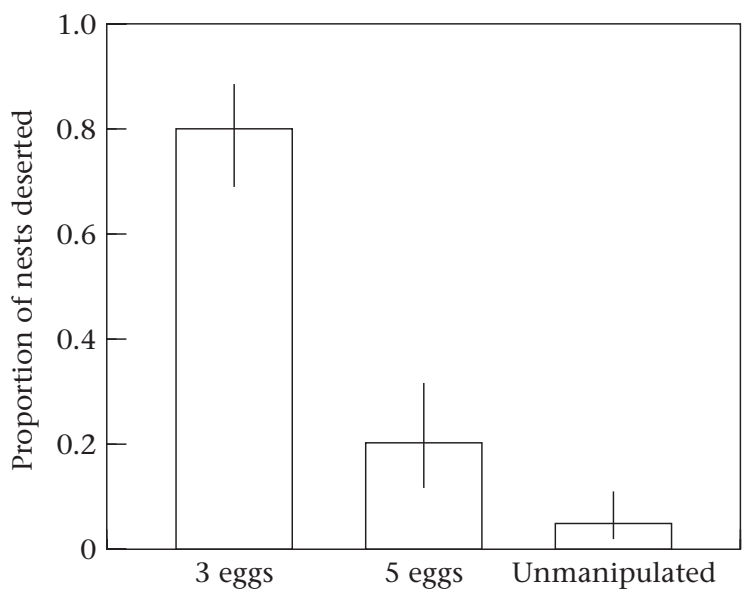

Figure 1. Proportion of great tit nests deserted and $95 \%$ confidence intervals for unmanipulated clutches $(N=104)$, experimentally reduced clutches with five eggs $(N=64)$ and with three eggs $(N=65)$. than unmanipulated clutches (chi-square test: $\chi_{1}^{2}=10.1$, $P=0.001$ ) and clutches reduced to three eggs were deserted more often than clutches reduced to five (chisquare test: $\left.\chi_{1}^{2}=50.4, P<0.001\right)$. We found that, controlled for manipulation category, the probability of nest desertion was positively related to the original clutch size (chi-square test: $\chi_{1}^{2}=6.12, P=0.01$ ). This indicated that females with large clutches were less likely to desert.

Thus, in accordance with the first prediction, the probability of clutch desertion was negatively related to the number of eggs that remained in the nest. However, the relative change in clutch size (i.e. the number of eggs removed) might have been important, rather than the actual number of eggs present in the nest.

\section{Prediction 2: Desertion and Incubation Stage}

Clutches reduced to five eggs on day 4 of incubation were more likely to be deserted than those reduced to five eggs on day 8. Similarly, pairs whose clutch was reduced to three eggs on day 8 were more likely to desert than those whose clutch was reduced to three eggs on day 12 (Fig. 2). Controlled for the number of eggs present after manipulation, desertion rates were significantly different at 4,8 and 12 days of incubation (chi-square test: $\left.\chi_{1}^{2}=24.7, P<0.001\right)$. The results are in line with the second prediction, that nest desertion rates should decrease with the stage of the breeding attempt at which the clutch is reduced.

To test the alternative hypothesis that accumulation of costs during the breeding attempt plays a role in the decision to desert the nest, we investigated whether there were indications for such costs. We compared replacement clutches of pairs of which all eggs had been removed after 4,8 or 12 days of incubation. All pairs whose clutch was removed $(N=32)$ produced a replacement clutch. Seven pairs renested in the extra nestbox 


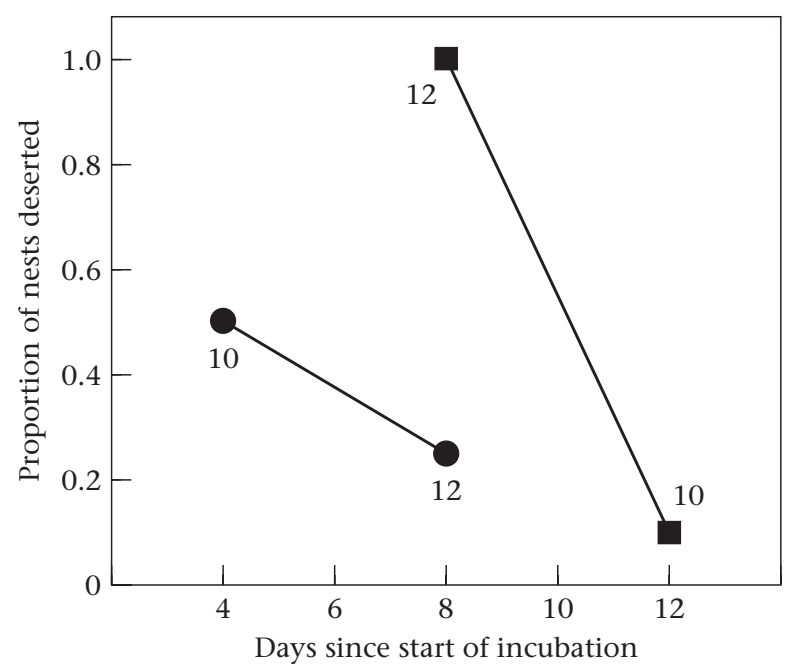

Figure 2. Proportion of great tit clutches deserted after clutch size reduction to three eggs $(\square)$ and to five eggs ( $)$, performed at different stages of the incubation period. The numbers indicate the sample sizes in each group (see also Table 1).

that we provided, another seven used the old nest and 18 renested in a nearby nestbox that was already present before the experiment. For two replacement clutches (one produced after 4 and one after 12 days of incubation) laying date, clutch size and egg volume were unknown.

The average length of the renesting interval differed between pairs that had their clutches removed after 4,8 and 12 days of incubation (mean 5.5, 5.6 and 7.3 days, respectively, ordered heterogeneity test: $r_{\mathrm{s}} P_{\mathrm{c}}=0.99, k=3$, $P<0.05$; Rice \& Gaines 1994). One pair was not included in this analysis, because it had an extremely long renesting interval (19 days) and we suspect that the female produced another replacement clutch which failed before she was identified (Dixon's test for outliers: $\alpha<0.05$ ). Both clutch size and mean egg volume were positively correlated between the replacement clutch and the first clutch (Fig. 3). Furthermore, eggs of individual females were on average larger in replacement clutches than in first clutches (mean difference $0.053 \mathrm{~cm}^{3}$, paired $t$ test: $\left.t_{27}=3.93, P<0.001\right)$. However, neither clutch size nor egg volume of replacement clutches following egg removal after 4,8 or 12 days of incubation was significantly different (ANOVA: clutch size: $F_{2,27}=0.51, P=0.5$; egg volume: $\left.F_{2,25}=2.10, P=0.14\right)$. From these comparisons we conclude that there is no clear evidence that the females incur a cost from spending more time incubating, although after 12 days of incubation the renesting interval seemed to be longer.

\section{Estimates of Fitness Consequences}

To compare the fitness consequences of continuing with a reduced clutch and deserting the current clutch in favour of future reproduction, we used the pairs with three eggs or five eggs supplemented with dummy eggs (Table 2).

The proportion of nests that produced at least one fledgling was not significantly different for birds that continued and birds that deserted (chi-square test: five eggs: $\chi_{1}^{2}=0.39, P=0.53$; three eggs: $\left.\chi_{1}^{2}=0.00, P=1.00\right)$. Fledging success was higher in reduced clutches than in replacement clutches (chi-square test: five eggs: $\chi_{1}^{2}=7.97$, $P=0.005$; three eggs: $\left.\chi_{1}^{2}=8.29, P=0.004\right)$. However, this did not compensate for the number of eggs removed and pairs that continued with a reduced clutch produced fewer fledglings than pairs with an experimentally induced replacement clutch (ANOVA: five eggs: $F_{1,18}=12.88, P=0.002$; three eggs: $\left.F_{1,17}=77.10, P<0.001\right)$. Furthermore, fledging mass was higher in reduced broods than in replacement broods (ANOVA: five eggs: $F_{1,18}=7.96, P=0.01$; three eggs: $\left.F_{1,17}=6.38, P=0.02\right)$.

The probability of a second clutch was higher for pairs with a reduced clutch than for pairs with a replacement clutch (chi-square test: five eggs: $\chi_{1}^{2}=9.29, P=0.002$; three eggs: $\chi_{1}^{2}=8.2, P=0.004$ ). Only one experimentally induced replacement brood was followed by a second clutch. As a consequence the number of fledglings produced over the entire breeding season was not significantly different between the birds that continued with a reduced first clutch and those with a replacement clutch (ANOVA: five eggs: $F_{1,21}=0.81, P=0.38$; three eggs: $F_{1,21}=0.00$, $P=0.94)$
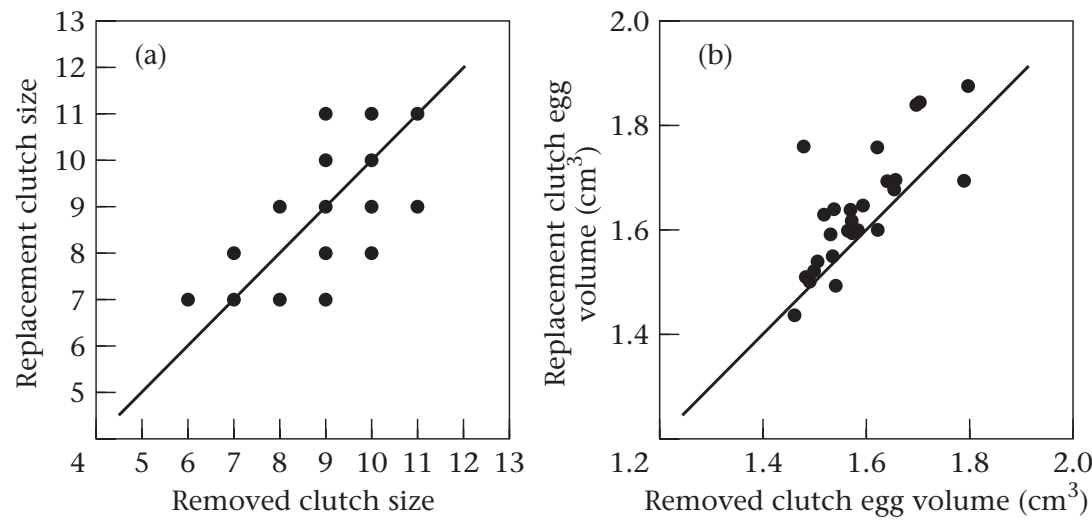

Figure 3. (a) Clutch size $\left(r_{28}=0.62, P<0.001\right)$ and (b) egg volume $\left(r_{26}=0.75, P=0.005\right)$ of first and experimentally induced replacement clutches of great tits. Ten clutch size combinations occurred more than once, involving 24 pairs. The lines indicate equal values for first and replacement clutches. 
Pairs that continued with a reduced clutch of three eggs produced fewer recruits in the first breeding attempt than pairs with a replacement brood (chi-square test: $\chi_{1}^{2}=3.96$, $P=0.05$ ). This was not the case for pairs with five-egg clutches (chi-square test: $\chi_{1}^{2}=0.21, P=0.65$ ). However, the total number of recruits produced over the entire breeding season was not significantly different between the two groups (chi-square test: five eggs: $\chi_{1}^{2}=0.29, P=0.59$; three eggs: $\chi_{1}^{2}=1.02, P=0.31$, including all pairs, irrespective of the success of the first brood). Survival of adult males (chi-square test: five eggs: $\chi_{1}^{2}=0.03, P=0.86$; three eggs: $\chi_{1}^{2}=0.63, P=0.43$ ) and adult females (chi-square test: five eggs: $\chi_{1}^{2}=0.69, P=0.41$; three eggs: $\chi_{1}^{2}=0.17, P=0.68$ ) did not differ between pairs that continued with a reduced clutch and pairs that deserted. However, samples sizes were small and, therefore, only large differences could have been detected.

In conclusion, we found no differences in adult survival and the number of fledglings or recruits measured over the total breeding season. Thus, fitness estimates of pairs that deserted and pairs that continued with a reduced clutch seemed to be similar.

\section{DISCUSSION}

Great tits responded to the clutch size reduction experiments by deserting their nest more frequently than unmanipulated pairs. The probability of clutch desertion was negatively related to the number of eggs that remained in the nest and positively to the original clutch size. Furthermore, nest desertion rates declined with the stage of the incubation period. Although there was a clear response of the birds to the size of the reduced clutch, we found no differences in the estimates of fitness consequences of the two alternative decisions (continue or desert).

\section{Clutch Size Before and After the Manipulation}

The probability of clutch desertion was negatively related to the size of the clutch after manipulation. Similar results were obtained by Winkel (1970), Armstrong \& Robertson (1988), Winkler (1991) and Delehanty \& Oring (1993) who experimentally reduced clutches to different sizes and found that desertion was more likely if only a few eggs remained.

The brood success hypothesis (Carlisle 1982) predicts higher levels of parental investment in successful than unsuccessful broods. For example, in common goldeneyes, Bucephala clangula, maternal effort was related to the mortality already experienced by the brood rather than brood size per se (Pöysä et al. 1997). Our results are in line with the brood success hypothesis, because clutch desertion was related not only to the number of eggs that remained in the nest, but also to the original clutch size, suggesting that the relative change in clutch size was important.

Furthermore, we found a positive correlation between the size of the replacement clutch and original clutch size (see also e.g. Arnold 1993). This suggests that the birds with a larger initial clutch were more likely to benefit from a replacement clutch. The decision to desert was thus not taken on the basis of manipulated clutch size alone. However, it remains unclear whether the birds decided on the basis of the number or the proportion of eggs that remained or disappeared or on the expected number of eggs in the replacement clutch. In our experiment these variables were correlated.

\section{Stage of the Breeding Attempt}

The probability of clutch desertion after egg removal decreased as the time of manipulation approached the end of the incubation period. Particularly in the group reduced to three eggs, there was a strong decrease from 100 to $10 \%$ over a period of only 4 days. Similar results were reported by Winkler (1991) who found that tree swallows, Tachycineta bicolor, deserted only if their broods were reduced early in the nesting cycle. These and our findings are consistent with the idea that nest desertion is related to the residual investments the parents have to make to finish the current breeding attempt and thus to the current value of the clutch.

Besides an increase in the value of the current brood relative to additional parental investment needed, accumulation of parental effort throughout the nesting cycle may also play a role in the decision to desert the nest. This is because accumulation of parental effort may increase the cost of producing a replacement clutch (Slagsvold 1984). In our experiment time spent incubating was correlated with the stage of the breeding attempt at which the eggs were removed. The fact that all birds whose clutch was removed produced a replacement clutch, that these clutches had bigger eggs (see also Slagsvold \& Lifjeld 1990; Verhulst et al. 1995) and that there were no differences in clutch size and egg volume between birds that had incubated for 4,8 or 12 days, suggests that the time spent incubating did not affect future reproduction. However, the interval between successive breeding attempts was positively related to the number of days the eggs had been incubated, a result found in several other species whose clutches or broods were removed (Wooller 1980; Fuchs 1981; Parker 1981; Swanson et al. 1986; but see Alliston 1979). Therefore, we cannot completely rule out the possibility that accumulation of costs during incubation partly accounted for different nest desertion probabilities at different stages of the breeding attempt.

\section{Fitness Estimates}

Several attempts have been made to predict when birds should desert a reduced brood, for example by calculating the cost of renesting in terms of time (Taborsky 1985; Townsend 1986; ten Cate \& Taborsky 1992) or offspring survival (Mock \& Parker 1986). Bauchau \& Seinen (1997) determined the 'optimal clutch size at desertion' as the number of eggs at which the expected value of the deserted clutch equalled that of the replacement clutch. However, in none of these studies were the fitness consequences of the two options (continue or desert) directly 
measured in a random sample of birds. In our study, we determined fitness estimates simultaneously and independently from nest desertion rates by using two separate sets of experiments.

Our fitness estimates of pairs that deserted or continued with a reduced clutch were not significantly different. If eggs were removed, the value of the first clutch was reduced. However, this was compensated by a higher frequency of second clutches in these groups. Clearly more data, including more years, are needed to reach firm conclusions on this point. The results suggest, however, that parents did not improve their fitness by deserting a reduced clutch. This is surprising, since we found high desertion rates in response to the experimental clutch size reductions.

One explanation for this discrepancy could be that females perceived a reduction in clutch size as predation. Females with an experimentally reduced clutch may desert, not because of a direct devaluation of the clutch, but because the predator may return and endanger the incubating female (Sonerud 1985). Nevertheless, predation risk explains the observed patterns of nest desertion only if it is a function of the number of eggs that remained in the nest or of the expected duration of future incubation. Perceived predation risk may depend on the number of eggs that disappear, but it seems unlikely that the fear for the return of a predator depends on the length of time the female has incubated. A number of species, including the great tit, avoid nestboxes or nest sites where predation has occurred previously (Harvey et al. 1979; Dow \& Fredga 1983; Sonerud 1985; Marjakangas et al. 1997). If an increase in perceived predation risk influences the decision to desert, females are not expected to use the same nestbox for a replacement clutch. In our study, great tits regularly laid the replacement clutch in the original box, even though an empty nestbox was always available.

Another possibility is that nest desertion behaviour is constrained by the physiological mechanism by which incubation is regulated. It is likely that tactile stimuli from the eggs and from the nest itself stimulate prolactin secretion, which in turn increases the readiness to incubate (Hall \& Goldsmith 1983). Thus, removing tactile stimuli by taking away eggs may result in nest desertion by lowering prolactin secretion in the incubating female. In all birds prolactin levels increase towards the end of the incubation period. Therefore, it is possible that clutch desertion is less likely to occur not only if more eggs remain in the nest, but also if egg removal takes place at a later stage of incubation.

The decision rules for nest desertion revealed in this study do not seem to maximize fitness. In this respect it is interesting that clutch size in this population was larger than optimal (Verhulst 1995). The absence of local adaptation of clutch size is possibly due to gene flow resulting from birds migrating from the mainland to the island of Vlieland (Verhulst \& van Eck 1996). Similarly nest desertion behaviour could be tuned to a global rather than to a local optimum. Although the general patterns of nest desertion behaviour identified in this study reflect a trade-off between current and future reproduction more data are needed to make quantitative predictions with regard to the fitness consequences of nest desertion.

\section{Acknowledgments}

Henk Sandee, Niels Klazenga, Peter Waanders, Frank Majoor, Hester van Griethuizen and Jan Visser collected data on breeding success of great tits and Irma de Groot, Ingrid van der Wal, Gerben van den Brink and Saskia van Rouveroy helped with the experiments. The late Huybert van Eck refurbished old nestboxes so that they could be used as temporary housing for renesting great tits. Staatsbosbeheer gave permission to work on Vlieland. We thank Marcel Visser for suggesting the use of dummy eggs and Vincent Bauchau, Kate Lessells, Arie van Noordwijk, Simon Verhulst, Marcel Visser, Tore Slagsvold and an anonymous referee for comments on the manuscript.

\section{References}

Alliston, W. G. 1979. Renesting by the redhead duck. Wildfowl, 30, 40-44.

Armstrong, T. \& Robertson, R. J. 1988. Parental investment based on clutch value: nest desertion in response to partial clutch loss in dabbling ducks. Animal Behaviour, 36, 941-943.

Arnold, T. W. 1993. Factors affecting renesting in American coots. Condor, 95, 273-281.

Bauchau, V. \& Seinen, I. 1997. Clutch desertion and renesting in the pied flycatcher: an experiment with progressive clutch removal. Animal Behaviour, 54, 53-161.

Beissinger, S. R. 1990. Experimental brood manipulations and the monoparental threshold in snail kites. American Naturalist, 136, 20-38.

Carlisle, T. R. 1982. Brood success in variable environments: implications for parental care allocation. Animal Behaviour, 30, 824-836.

Crawley, M. J. 1993. GLIM for Ecologists. Oxford: Blackwell Scientific.

Daan, S., Dijkstra, C. \& Tinbergen, J. M. 1990. Family planning in the kestrel (Falco tinnunculus): the ultimate control of covariance of laying date and clutch size. Behaviour, 114, 83-116.

Delehanty, D. J. \& Oring, L. W. 1993. Effect of clutch size on incubation persistence in male Wilson's phalaropes (Phalaropes tricolor). Auk, 110, 521-528.

Dow, H. \& Fredga, S. 1983. Breeding and natal dispersal of the goldeneye, Bucephala clangula. Journal of Animal Ecology, 52, 681-695.

Fernández, G. J. \& Reboreda, J. C. 2000. Egg losses and nest desertion in greater rheas Rhea americana. Ibis, 142, 29-34.

Fuchs, S. 1981. Consequences of premature weaning on the reproduction of mothers and offspring in laboratory mice. Zeitschrift für Tierpsychologie, 55, 19-32.

Hall, M. R. \& Goldsmith, A. R. 1983. Factors affecting prolactin secretion during breeding and incubation in the domestic duck (Anas platyrhynchos). General Comparative Endocrinology, 49, 270-276.

Harvey, P. H., Greenwood, P. J. \& Perrins, C. M. 1979. Breeding area fidelity of great tits (Parus major). Journal of Animal Ecology, 48, 305-313.

Kluyver, H. N. 1951. The population ecology of the great tit Parus m. major. Ardea, 39, 1-135.

Kluyver, H. N. 1971. Regulation of numbers in populations of great tits (Parus m. major). In: Dynamics of Numbers in Populations (Ed. by P. J. den Boer \& G. R. Gradwell), pp. 507-523. Wageningen: Pudoc. 
Lessells, C. M. 1993. The cost of reproduction: do experimental manipulations measure the edge of the options set? Etología, 3, 95-111.

Marjakangas, A., Valkeajärvi, P. \& ljäs, L. 1997. Female black grouse Tetrao tetrix shift nest site after nest loss. Journal für Ornithologie, 138, 111-116.

Mock, D. W. \& Parker, G. A. 1986. Advantages and disadvantages of egret and heron brood reduction. Evolution, 40, 459-470.

van Noordwijk, A. J., Keizer, L. C. P., van Balen, J. H. \& Scharloo, W. 1981. Genetic variation in egg dimensions in a population of great tits. Genetica, 55, 221-232.

Numerical Algorithms Group 1993. GLIM Statistical Package. Version 4. Oxford: Numerical Algorithms Group.

Parker, H. 1981. Renesting biology of Norwegian willow ptarmigan. Journal of Wildlife Management, 45, 858-864.

Pettifor, R. A., Perrins, C. M. \& McCleery, R. H. 1988. Individual optimization of clutch size in great tits. Nature, 336, 160-162.

Pöysä, H., Virtanen, J. \& Milonoff, M. 1997. Common goldeneyes adjust maternal effort in relation to prior brood success and not current brood size. Behavioral Ecology and Sociobiology, 40, 101-106

Rice, W. R. \& Gaines, S. D. 1994. Extending nondirectional heterogeneity tests to evaluate simply ordered alternative hypotheses. Proceedings of the National Academy of Sciences U.S.A., 91, 225226.

Slagsvold, T. 1982. Clutch size variation in passerine birds: the nest predation hypothesis. Oecologia, 54, 159-169.

Slagsvold, T. 1984. Clutch size variation of birds in relation to nest predation: on the cost of reproduction. Journal of Animal Ecology, 53, 945-953.

Slagsvold, T. \& Lifjeld, J. T. 1990. Influence of male and female quality on clutch size in tits (Parus spp.). Ecology, 71, 1258-1266.

Sokal, R. R. \& Rohlf, F. J. 1995. Biometry. New York: W. H. Freeman.

Sonerud, G. A. 1985. Nest hole shift in Tengmalm's owl Aegolius funereus as defence against nest predation involving long-term memory in the predator. Journal of Animal Ecology, 54, 179-192.

Swanson, G. A., Shaffer, T. L., Wolf, J. F. \& Lee, F. B. 1986. Renesting characteristics of captive mallards on experimental ponds. Journal of Wildlife Management, 50, 32-38.

Taborsky, M. 1985. On optimal paternal care. Zeitschrift für Tierpsychologie, 70, 331-336.

ten Cate, C. \& Taborsky, M. 1992. To raise or to abandon a reduced clutch: a theoretical approach illustrated using ringed turtle-doves (Streptopelia risoria). Auk, 109, 594-600.

Tinbergen, J. M. \& Daan, S. 1990. Family planning in the great tit (Parus major): optimal clutch size as integration of parent and offspring fitness. Behaviour, 114, 161-190.

Townsend, D. S. 1986. The costs of male parental care and its evolution in a neotropical frog. Behavioural Ecology and Sociobiology, 19, 187-195.

Verhulst, S. 1995. Reproductive decisions in the great tit; an optimality approach. Ph.D. thesis, University of Groningen.

Verhulst, S. \& van Eck, H. M. 1996. Gene flow and immigration in an island population of great tits. Journal of Evolutionary Biology, 9, 771-782.

Verhulst, S., van Balen, J. H. \& Tinbergen, J. M. 1995. Seasonal decline in reproductive success of the great tit: variation in time or quality. Ecology, 76, 2392-2403.

Williams, G. C. 1966. Natural selection, the costs of reproduction, and a refinement of Lack's principle. American Naturalist, 100, 687-690.

Winkel, W. 1970. Wie reagiert der Feldsperling (Passer montanus) auf die Fortnahme bebrüteter Eier? Die Vogelwelt, 91, 243245.

Winkler, D. W. 1991. Parental investment decision rules in tree swallows: parental defense, abandonment, and the so-called Concorde Fallacy. Behavioral Ecology, 2, 133-142.

Wooller, R. D. 1980. Repeat laying by kittiwakes Rissa tridactyla. Ibis, 122, 226-229. 